\title{
CRESCIMENTO E TOLERÂNCIA DE MUDAS DE Enterolobium contortisiliquum Vell. CULTIVADAS EM SOLO CONTAMINADO COM ZINCO
}

\section{GROWTH AND TOLERANCE OF Enterolobium contortisiliquum Vell. SEEDLINGS GROWN IN CONTAMINATED SOIL WITH ZINC}

\author{
Rodrigo Ferreira da Silva ${ }^{1}$ Clovis Orlando Da $\operatorname{Ros}^{1}$ Zaida Ines Antoniolli ${ }^{2}$ André Luis Grolli ${ }^{3}$ Douglas \\ Leandro Scheid ${ }^{4}$ Gilvan Moises Bertollo ${ }^{4}$ Evandro Luis Missio ${ }^{5}$
}

\section{RESUMO}

O zinco é um elemento essencial às plantas, mas quando presente em altas concentrações no solo pode se tornar tóxico ao metabolismo vegetal. $\mathrm{O}$ trabalho objetivou determinar o crescimento e tolerância de mudas de Enterolobium contortisiliquum Vell. cultivadas em solo contaminado com zinco. O experimento foi conduzido em casa de vegetação, em solo com $81 \%$ de argila, no delineamento inteiramente casualizado, com sete tratamentos, constituídos de doses de zinco $\left(0,100,200,300,400,500\right.$ e $\left.600 \mathrm{mg} \mathrm{kg}^{-1}\right)$ e oito repetições. Avaliou-se a altura de planta, diâmetro de colo, massa seca aérea, massa seca radicular, comprimento da raiz principal, área superficial específica, teor de Zn na parte aérea e radicular, coeficiente do impacto do teor relativo de zinco, índice de translocação e de tolerância. O coeficiente do impacto do teor relativo de zinco indicou tendência de menor translocação de $\mathrm{Zn}$ à parte aérea da muda até a dose de $300 \mathrm{mg}$ de $\mathrm{Zn} \mathrm{kg}^{-1}$ de solo. Os resultados evidenciaram que doses próximas a $200 \mathrm{mg}$ de zinco $\mathrm{kg}^{-1}$ de solo argiloso estimulam a altura de planta, diâmetro do colo, a massa seca aérea e radicular das mudas de timbaúva. A timbaúva apresenta elevada tolerância ao zinco e por ser uma arbórea nativa adaptada às condições ambientais do Brasil pode ser indicada para revegetação de áreas contaminadas com a aplicação de até $600 \mathrm{mg}$ de zinco $\mathrm{kg}^{-1}$ de solo argiloso.

Palavras-chave: metais; fitorremediação; espécies arbóreas.

\begin{abstract}
Zinc is an essential element to plants, but when present in high concentrations in the soil can become toxic to the plant metabolism. The study aimed to determine the growth and tolerance of Enterolobium contortisiliquum Vell. seedlings cultived in soil contaminated with zinc. The experiment was conducted in a greenhouse in soil with $81 \%$ clay, in a completely randomized design with seven treatments, doses of zinc $\left(0,100,200,300,400,500\right.$ and $\left.600 \mathrm{mg} \mathrm{kg}^{-1}\right)$ eight repetitions. We evaluated the plant height, stem diameter, shoot dry mass, root dry mass, length of the main root, specific surface area, $\mathrm{Zn}$ content in shoots and roots, the impact coefficient of relative zinc content, translocation and tolerance. The coefficient of impact of the relative zinc content indicated a trend of lower translocation of $\mathrm{Zn}$ to the aerial part of the molt up to the dose of $300 \mathrm{mg}$ of $\mathrm{Zn} \mathrm{kg-1}$ of soil. The results showed that doses close to $200 \mathrm{mg} \mathrm{Zinc} \mathrm{kg} \mathrm{clay}^{-1}$ soil stimulate plant height, stem diameter, stem and root dry mass of seedlings and timbaúva. Timbaúva

1 Eng. Agrônomo, Dr., Professor da Universidade Federal de Santa Maria, Br 386, Km 40, Linha 7 de setembro, s/n, CEP: 98400-000, Frederico Westphalen (RS), Brasil.rodrigosilva@smail.ufsm.br; clovisdaros@gmail.com

2 Bióloga, Dr ${ }^{\mathrm{a}}$., Professor do Departamento de Solo, Centro de Ciências Rurais, Universidade Federal de Santa Maria, Av. Roraima, 1000, CEP 97105-900, Santa Maria (RS), Brasil. zantoniolli@gmail.com

3 Eng. Agrônomo, Mestre em Agronomia: Agricultura e Ambiente, UFSM, Br 386, Km 40, Linha 7 de setembro, s/n, CEP: 98400-000, Frederico Westphalen (RS), Brasil. andregrolli1990@hotmail.com

4 Eng. Agrônomo, Doutorandos nos Programas de Pós-Graduação em Ciência do Solo e em Engenharia Agrícola, Centro de Ciências Rurais, Universidade Federal de Santa Maria, Av. Roraima, 1000, CEP 97105-900, Santa Maria (RS), Brasil. douglasscheid@gmail.com; gilvanbertollo@yahoo.com.br

5 Eng. Agrônomo, Dr., Pesquisador do Departamento de Diagnóstico e Pesquisa Agropecuária (DDPA/SEAPI), Distrito de Boca-do-Monte, Km 4,5, CEP 97170-000, Santa Maria (RS), Brasil. evandro@seapi.rs.gov.br
\end{abstract}

Recebido para publicação em 26/09/2014 e aceito em 26/06/2017 
presents high tolerance to zinc and because it is a native tree adapted to Brazilian environmental conditions it can be indicated for revegetation of contaminated areas with the application of up to $600 \mathrm{mg}$ of zinc $\mathrm{kg}-1$ of clay soil.

Keyword: metals; phytoremediation; tree species.

\section{INTRODUÇÃO}

O Zn pode ser encontrado em vários resíduos ou rejeitos produzidos em elevada quantidade pela população (LUXRESEARCH, 2009), como nos descartes de equipamentos eletrônicos (INTERNATIONAL SOLID WASTE ASSOCIATION, 2012), dejeto liquido de suíno (BASSO et al., 2012), lodo de esgoto e biossólidos (KABATA-PENDIAS, 2010) e rejeitos de mineração (BORGES JUNIOR et al., 2008). Além disso, tem-se evidenciado que a aplicação sucessiva e de modo contínuo por 10 anos de fungicidas contendo cobre e zinco acarreta em aumento dos elementos na camada superficial do solo (BRUNETTO et al., 2014). Desse modo, diariamente, diferentes fontes de zinco podem estar atingindo o solo possibilitando a elevação de suas concentrações.

$\mathrm{O} \mathrm{Zn}$, embora seja considerado um elemento essencial para o desenvolvimento das plantas, absorvido da solução do solo como $\mathrm{Zn}^{2+}$ (SANTOS et al., 2011), pode se tornar extremamente fitotóxico (ROUT; DIAS, 2009). Isso ocorre quando suas concentrações estão entre 100 a $500 \mathrm{mg} \mathrm{Zn} \mathrm{kg}{ }^{-1}$ de solo (KABATA-PENDIAS, 2010). Nesse sentido, o Conselho Nacional do Meio Ambiente estabeleceu, por meio da resolução $n^{\circ} 420$ (CONSELHO NACIONAL DO MEIO AMBIENTE, 2009), o limite de 450 $\mathrm{mg}$ de $\mathrm{Zn} \mathrm{kg}^{-1}$ de solo como o valor de investigação para solos agrícolas, pois, acima desta concentração, esse elemento promove redução do crescimento vegetal. Sintomas de toxicidade do $\mathrm{Zn}$ às plantas incluem deficiência induzida de $\mathrm{Fe}$, levando à clorose pela diminuição da síntese de clorofila e degradação dos cloroplastos, além de interferir na absorção de fósforo $(\mathrm{P})$, magnésio $(\mathrm{Mg})$ e manganês $(\mathrm{Mn})$ (BROADLEY et al., 2007). O estudo de plantas que tolerem os teores em excesso desse metal no solo torna-se importante para possibilitar a revegetação de áreas contaminadas.

As plantas apresentam mecanismos de tolerância aos metais pesados, como a alteração na atividade das enzimas antioxidantes (catalase e superóxido dismutase), a complexação do metal em compostos intracelulares (fitoquelatinas e metalotioninas) e a compartimentalização no vacúolo (KRÄMER et al., 2000). Desse modo, as plantas podem ser classificadas conforme sua resposta à concentração de metais no solo, em exclusoras, indicadoras e acumuladoras (SALT, 2001). Entretanto, ainda é escassa a relação de espécies arbóreas nativas tropicais que reconhecidamente apresentam desenvolvimento em solos contaminados com zinco.

Diversos trabalhos de pesquisa indicam efeito positivo da utilização de plantas em solos contaminados com zinco, sejam espécies florestais, como evidenciado para Corymbia citriodora tolerante à adição de até $1.500 \mathrm{mg} \mathrm{kg}^{-1}$ de zinco em solo com $81 \%$ de argila (SILVA et al., 2016), para Cordia africana na fitoestabilização de Zn (PEREIRA et al., 2013), para mogno-africano ( $K$. senegalensis A. Juss) que tolera apenas $20 \mathrm{mg}$ de zinco $\mathrm{dm}^{-3}$ (ARAÚJO et al., 2017), ou agronômicas como o Zea mays indicado para fitorremediar zinco e cádmio (VAN SLYCKEN et al., 2010). Contudo, a literatura não relata a influência da contaminação do solo com zinco no crescimento de mudas de timbaúva (Enterolobium contortisiliquum Vell.) que é uma espécie arbórea nativa pioneira, pertencente à família Leguminosae, de ocorrência nos Estados do Mato Grosso e do Ceará até o Rio Grande do sul (GRINGS; BRACK, 2011). Esta planta apresenta tendência de armazenamento de cobre nas raízes e baixa translocação para a parte aérea, resultando em incremento na massa seca da parte aérea com pequenas doses de cobre no solo (SILVA et al., 2011). Nesse sentido, uma questão ainda persiste sobre a possibilidade da timbaúva crescer e ser tolerante a solo contaminado com Zn. Desse modo, este trabalho objetivou determinar o crescimento e a tolerância de mudas de Enterolobium contortisiliquum Vell. cultivadas em solo contaminado com zinco.

\section{MATERIAL E MÉTODOS}

O experimento foi conduzido em casa de vegetação pertencente ao Departamento de Ciências Agronômicas e Ambientais da UFSM, Campus de Frederico Westphalen, Rio Grande do Sul, Brasil. 
O solo utilizado foi classificado como Latossolo Vermelho (EMBRAPA, 2013), cuja caracterização química e física está apresentada na Tabela 1.

TABELA 1: Caracterização química e física do solo utilizado como substrato para a produção de mudas de timbaúva em casa de vegetação. Frederico Westphalen-RS, 2014.

TABLE 1: Chemical and physical soil used as substrate for the production of seedlings in the greenhouse timbaúva characterization. Frederico Westphalen, RS state, 2014.

\begin{tabular}{|c|c|c|c|c|c|c|c|c|}
\hline $\begin{array}{c}\text { pH água } \\
(1: 1)\end{array}$ & $\mathrm{Ca}+\mathrm{Mg}$ & $\mathrm{Al}$ & $\mathrm{H}+\mathrm{Al}$ & $\mathrm{P}$ & $\mathrm{K}$ & $\mathrm{Zn}$ & $\mathrm{MO}$ & Argila \\
\hline & \multicolumn{3}{|c|}{----- $\mathrm{Cmol}_{\mathrm{c}} \mathrm{dm}^{-3}$} & \multicolumn{3}{|c|}{------- $\mathrm{mg} \mathrm{dm}^{-3}$------ } & \multicolumn{2}{|c|}{------- \% ------ } \\
\hline 5,5 & 5,4 & 0,4 & 4,3 & 6,6 & 110 & 1,1 & 2,4 & 81 \\
\hline
\end{tabular}

As unidades experimentais (EU) foram compostas por recipientes plásticos de $125 \mathrm{~cm}^{3}$ contendo uma planta. A espécie arbórea utilizada foi a timbaúva (Enterolobium contortisiliquum Vell.) cujas sementes foram provenientes da FEPAGRO Florestas - Centro de Pesquisas Florestais, unidade de Santa Maria-RS. As sementes foram submetidas à quebra de dormência por escarificação mecânica, sendo semeadas três sementes por UE e, quando as plântulas apresentaram um par de folhas definitivas foi realizado o desbaste, deixando-se uma planta por unidade experimental.

O experimento foi conduzido por 120 dias, com três irrigações diárias mantendo o solo na capacidade de campo. As fertilizações foram realizadas na pré-semeadura com $150 \mathrm{~g}$ de N, $300 \mathrm{~g}$ de $\mathrm{P}_{2} \mathrm{O}_{5}$ e $100 \mathrm{~g}$ de $\mathrm{K}_{2} \mathrm{O}$ por $\mathrm{m}^{3}$ de substrato e, em pós-semeadura, utilizaram-se $200 \mathrm{~g}$ de $\mathrm{N}$ e $150 \mathrm{~g}$ de $\mathrm{K}_{2} \mathrm{O}$ em solução, diluídos em $100 \mathrm{~L}$ de água, aos 30 dias após a germinação sendo aplicado $\mathrm{N}$ e K, aos 60 dias após a emergência apenas $\mathrm{N}$ e aos 90 dias após a germinação, $\mathrm{N}$ e $\mathrm{K}$ seguindo as recomendações de Gonçalves \& Benedetti (2005).

O delineamento experimental foi inteiramente casualizado com 7 tratamentos compostos de doses de zinco, sendo: 0 (controle sem adição de zinco), 100, 200, 300, 400, 500 e $600 \mathrm{mg}$ de Zn kg-1 de solo, com oito repetições. O solo foi contaminado com a adição de solução de sulfato de zinco $\left(\mathrm{ZnSO}_{4} 7 \mathrm{H}_{2} \mathrm{O}\right)$.

Os parâmetros avaliados foram: altura da parte aérea (AP), medida com régua graduada do colo da planta até o meristema apical, diâmetro do caule (DC) obtido com paquímetro digital pela medida na região do colo da planta, peso da massa seca da parte aérea (MSPA), peso da massa seca da parte raiz (MSPR), peso da massa seca total (PMST), comprimento de raiz principal (CRP) e área superficial específica (ASE), conforme Tennant (1975), teor de $\mathrm{Zn}$ na raiz e parte aérea. As plantas foram seccionadas na região do colo, separando-se a parte aérea da radicular, as quais foram submetidas à secagem em estufa a $60^{\circ} \mathrm{C} \pm$ $1^{\circ} \mathrm{C}$, até peso constante, sendo a massa seca da parte aérea e radicular quantificadas em balança analítica. A análise química do tecido vegetal foi realizada por digestão úmida com $\mathrm{HNO}_{3}+\mathrm{HClO}_{4}(3: 1)$ e posterior determinação do teor zinco da parte aérea $(\mathrm{TZnA})$ e radicular $(\mathrm{TZnR})$ por espectrofotometria de absorção atômica com chama de ar acetileno (EMBRAPA, 2009). Utilizando-se os valores dos parâmetros avaliados, na dose zero e na maior dose $\left(600 \mathrm{mg} \mathrm{kg}^{-1}\right)$, calculou-se o percentual de redução ou aumento (PRA) pela fórmula ((dose 600 / dose 0$)-1)$ x 100.

Calculou-se também o índice de tolerância, o coeficiente de impacto do teor relativo de zinco e o índice de translocação de zinco. O índice de tolerância (IT) foi calculado pela fórmula: [(PMST contaminado/ PMST controle) X 100], sendo PMST contaminado, o peso da massa seca total da muda crescida em solo contaminado e PMST controle, o peso da massa seca total da muda crescida no controle sem adição de $\mathrm{Zn}$ (ZACCHINI et al., 2009). O coeficiente de impacto do teor relativo de zinco (CITR) foi calculado com base na relação entre o teor na raiz e na parte aérea na dose zero $\left(\mathrm{R}_{0} / \mathrm{PA}_{0}\right)$ e nas doses com zinco $\left(\mathrm{R}_{\mathrm{Zn}} / \mathrm{PA}_{\mathrm{Zn}}\right)$, utilizando-se a fórmula CITR $=\left(\mathrm{R}_{\mathrm{Zn}} / \mathrm{PA}_{\mathrm{Zn}}\right) /\left(\mathrm{R}_{0} / \mathrm{PA}_{0}\right)$. O índice de translocação de zinco (ITZn), expresso em percentagem, foi calculado pela fórmula ITZn $=(\mathrm{CPAZn} / \mathrm{CTZn}) \times 100($ ABICHEQUIER; BOHNEN, 1998), sendo CPAZn o conteúdo de zinco acumulado na parte aérea e CTZn o total na planta. O conteúdo de $\mathrm{Zn}$ foi obtido por meio da fórmula: $\mathrm{CZn}=$ Teor $\mathrm{Zn}\left(\mathrm{mg} \mathrm{kg}^{-1}\right)$ x Massa Seca $(\mathrm{g})$.

Os resultados foram submetidos à análise de variância, com probabilidade de erro de 5\% 
$(\mathrm{p} \leq 0,05)$, quando significativo foram ajustadas equações de regressão, através do programa estatístico SISVAR (FERREIRA, 2011). Os resultados do índice de tolerância e do coeficiente de impacto do teor relativo de zinco, por serem relativos ao controle foram comparados pelo teste de Tukey a $5 \%$ de probabilidade de erro $(\mathrm{p}<0,05)$.

\section{RESULTADOS E DISCUSSÕES}

A análise de regressão revelou alterações significativas $(\mathrm{p} \leq 0,05)$ nos parâmetros avaliados em função das doses de $\mathrm{Zn}$ (Figura 1). Observou-se aumento na altura, diâmetro de colo das mudas de timbaúva, atingindo ponto de máxima com 232 e 212,5 $\mathrm{mg}$ de $\mathrm{Zn} \mathrm{kg}^{-1} \mathrm{de}$ solo, respectivamente, porém, com redução de 23,3 e 19,8\% na maior dose de $\mathrm{Zn}$ em relação à dose zero (Figuras 1A e 1B). O Zn é um elemento essencial para o desenvolvimento das plantas, por estar presente em seis classes de enzimas, desempenhando funções estruturais ou como catalizador (BROADLEY et al., 2007; HOODA, 2010). Desse modo, essas doses iniciais possibilitaram teores de suficiência de $\mathrm{Zn}$ no solo para o crescimento em altura e diâmetro das mudas de timbaúva e refletem a necessidade de estudos com ênfase na fertilização com micronutrientes, para maximizar o crescimento das espécies arbóreas nativas.

$\mathrm{Na}$ massa seca da parte aérea e radicular observou-se ponto de máxima em 187 e $200 \mathrm{mg} \mathrm{kg}^{-1} \mathrm{de}$ Zn, respectivamente (Figuras 1C e 1D). Zeitouni, Berton e Abreu (2007) observaram diminuição na matéria seca das plantas de mamona com o aumento das doses dos metais aplicadas ao solo. Sousa (2010) também evidenciou redução no crescimento das mudas de Mimosa caesalpiniaefolia Benth com o aumento das doses de zinco. Nesse sentido, Pavlíkováa et al. (2008) evidenciaram que a absorção de alta concentração de $\mathrm{Zn}$ de um solo contaminado, afeta o funcionamento da rubisco, reduzindo a produção de biomassa, em plantas de espinafre. Desse modo, é possível que a timbaúva tenha sua atividade enzimática afetada somente a partir de $187 \mathrm{mg}$ de $\mathrm{Zn} \mathrm{kg}^{-1}$ de solo contendo $81 \%$ de argila, mas essa hipótese necessita ser comprovada em outros trabalhos, para arbóreas nativas.
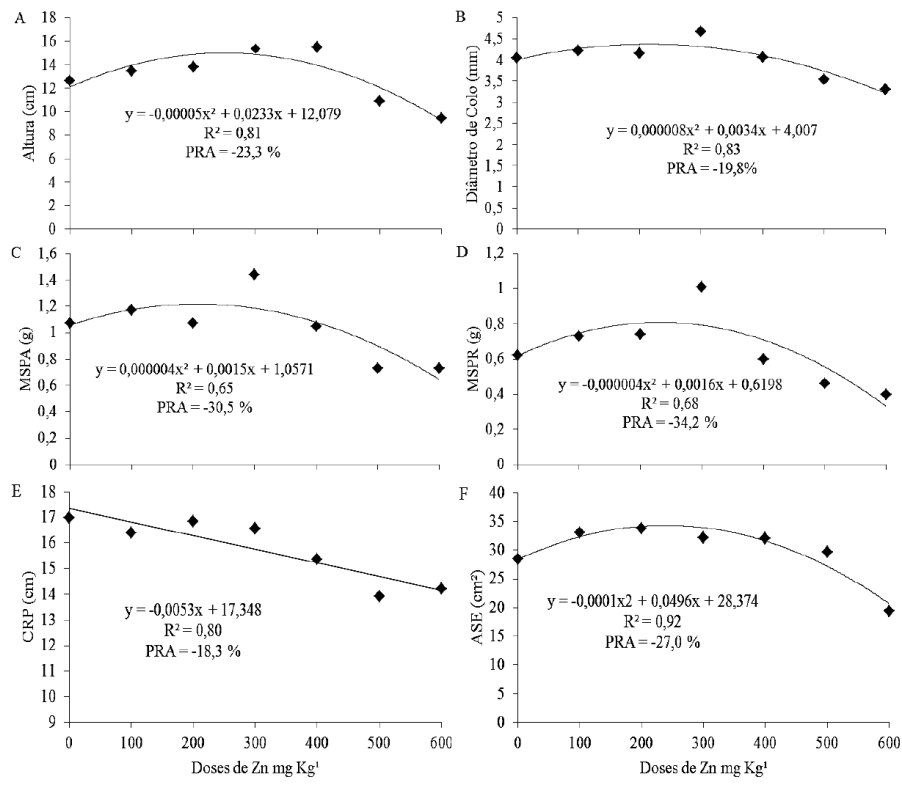

FIGURA 1: Equações de regressão para a altura (A), diâmetro de colo (B) massa seca da parte aérea - MSA (C), massa seca da parte raiz - MSR (D), comprimento da raiz principal - CRP (E) área superficial específica - ASE (F) das mudas de Enterolobium contortisiliquum submetidas a doses de cobre no solo. Frederico Westphalen-RS, 2014.

FIGURE 1: Regression equations for height (A), stem diameter (B) Dry weight of shoot - MSA (C), root dry mass arial part - MSR (D), length of the main root - CRP (E) specific surface area - ASE (F) of seedlings Enterolobium contortisiliquum subjected to doses of copper in the soil. Frederico Westphalen, RS state, 2014. 
O comprimento da raiz principal diminuiu linearmente com o aumento das doses de $\mathrm{Zn}$ no solo e a área superficial específica radicular aumentou significativamente em doses iniciais, com ponto de máxima em 245,0 $\mathrm{mg} \mathrm{kg}^{-1}$ (Figuras 1E e 1F). O excesso de $\mathrm{Zn}$ no solo geralmente reduz o comprimento radicular (HOODA, 2010), pois pode causar danos aos processos fisiológicos, afetando a mitose e causando a morte celular (EL-GHAMERY; EL-KHOLY; ABOU EL-YOUSSER, 2003). Soares et al. (2001) verificaram que as doses de $1.200 \mathrm{e} 1.600 \mu \mathrm{mol} \mathrm{L}{ }^{-1}$ de Zn causaram morte das plantas de Eucalyptus maculata, cultivado em solução nutritiva e, em concentrações menores, as plantas apresentaram raízes escuras e pouco desenvolvidas. Isso indica que sob estresse, induzido pelas doses de zinco, as mudas de timbaúva inicialmente emitem raízes novas e finas, mas, em doses maiores há um engrossamento e menor produção de raízes, o que pode ser evidenciado neste trabalho pela redução de $18,3 \%$ no comprimento da raiz principal e $27 \%$ na área superficial específica radicular na dose mais elevada em relação à dose zero (Figuras 1E e 1F).

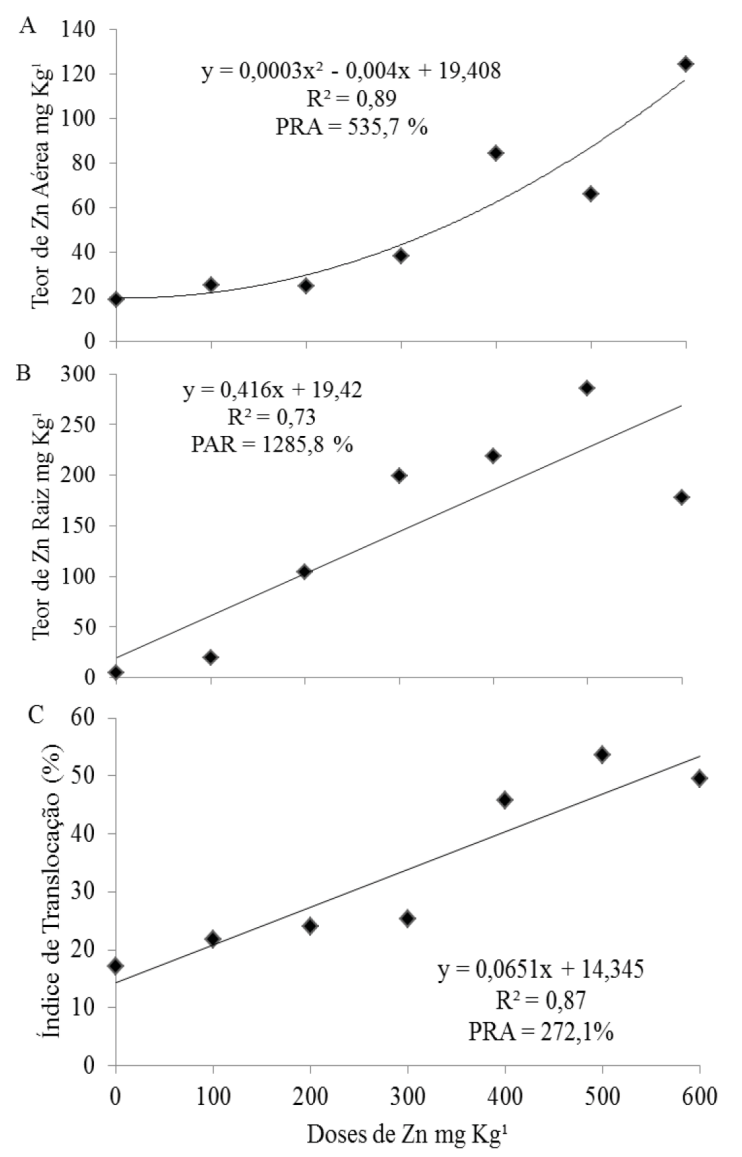

FIGURA 2: Equações de regressão para o teor de zinco na parte aérea (A), teor de zinco na raiz (B) e índice de translocação (C) das mudas de Enterolobium contortisiliquum submetidas a doses de cobre no solo. Frederico Westphalen-RS, 2014.

FIGURE 2: Regression equations for the zinc content in shoots (A), zinc content in the root (B) and Index of translocation (C) of Enterolobium contortisiliquum seedlings subjected to doses of copper in the soil. Frederico Westphalen, RS state, 2014.

Os teores de $\mathrm{Zn}$ na parte aérea e radicular aumentaram 535,7 e 1.285,8\% na maior dose, respectivamente, comparadas à testemunha, além disso, há maior teor de $\mathrm{Zn}$ na parte radicular com o aumento das doses (Figuras 2A e 2B). Isso se deve ao $\mathrm{Zn}$ ser de fácil translocação dos tecidos radiculares para os tecidos aéreos da planta (PULFORD; WATSON, 2003). Entretanto, Testiati et al. (2013) trabalhando com Globularia alypum L. e Rosmarinus officinalis L. em solo contaminado com zinco, encontraram teores elevados de zinco tanto na parte aérea, quanto nas raízes. Desse modo, esses resultados indicam a 
possibilidade da timbaúva ser uma espécie capaz de acumular Zn nos seus tecidos radiculares.

O Índice de Translocação de zinco das mudas de timbaúva apresentou resposta linear positiva (Figura 2C). Esse Resultado é semelhante aos encontrados por Rajoo et al. (2013) trabalhando com Dipterocarpus verrucosus em solo com aplicação de lodo de esgoto com altas concentrações de zinco. O crescimento de espécies arbóreas em solos contaminados com $\mathrm{Zn}$ relaciona-se com a capacidade de acumular ou translocar o metal (MARQUES; MOREIRA; SIQUEIRA, 2000). As mudas de timbaúva absorvem e translocam zinco para a parte aérea, além de acumular grande quantidade deste elemento em suas raízes.

A timbaúva apresentou maior índice de tolerância na dose de $300 \mathrm{mg} \mathrm{kg}^{1}$ de $\mathrm{Zn}$ no solo (Figura 3A). Quando este índice for maior que 60\% tem-se alta tolerância das plantas aos contaminantes (LUX et al., 2004). Desse modo, a timbaúva por apresentar valores superiores a $70 \%$ em todas as doses testadas, pode ser uma planta promissora para cultivo em solos argilosos contaminados com até $600 \mathrm{mg} \mathrm{kg}^{-1} \mathrm{de} \mathrm{zinco.}$
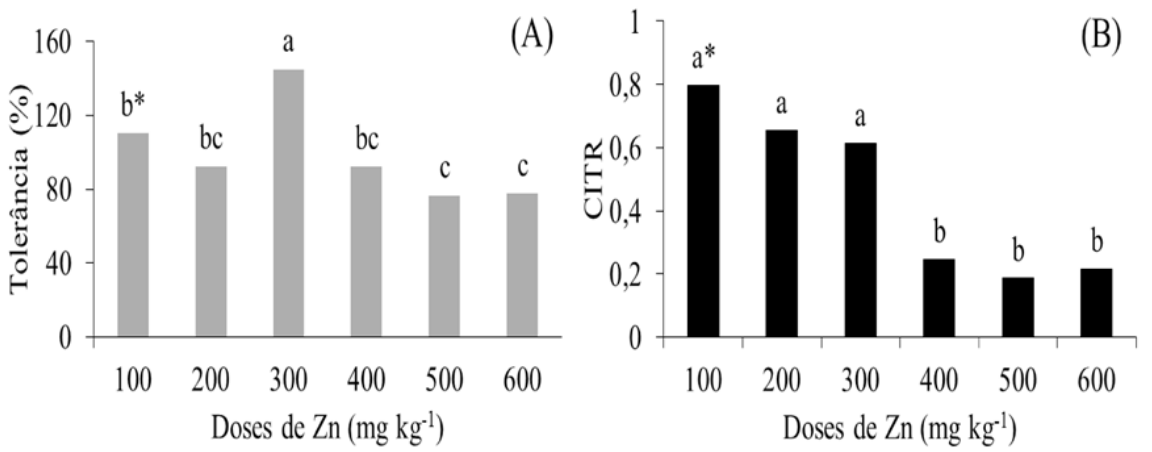

FIGURA 3: Índice de Tolerância (A) e Coeficiente de Impacto do Teor Relativo de Zinco - CITR (B) das mudas de Enterolobium contortisiliquum submetidas a doses de zinco no solo. Frederico Westphalen-RS, 2014. *Médias seguidas da mesma letra maiúscula, não diferem entre si pelo teste de Tukey a $5 \%$ de probabilidade de erro $(\mathrm{p}<0,05)$.

FIGURE 3: Index of Tolerance (A) and Coefficient of Relative Impact of Zinc Content - CITR (B) of Enterolobium contortisiliquum seedlings subjected to doses of zinc in the soil. Frederico Westphalen, RS state, 2014. * Means followed by the same capital letter do not differ by Tukey test at $5 \%$ level of probability (p $<0.05$ ).

O coeficiente de impacto do teor relativo foi significativamente menor a partir de $400 \mathrm{mg} \mathrm{kg}{ }^{1}$ de Zn no solo (Figura 3B). Quando o CITR é maior que uma unidade indica que mais metal é retido nas raízes da muda cultivada em solo contaminado (MARQUES; MOREIRA; SIQUEIRA, 2000). Assim, a tolerância apresentada pela timbaúva deve-se a uma tendência de menor translocação de $\mathrm{Zn}$ à parte aérea até a dose de $300 \mathrm{mg} \mathrm{kg}^{-1}$ de $\mathrm{Zn}$ no solo, o que reduz o impacto na fisiologia da planta e permite que ocorra desenvolvimento das mudas. Porém, nas doses de 400, 500 e $600 \mathrm{mg} \mathrm{kg}^{-1}$ de $\mathrm{Zn}$ no solo ocorreram valores baixos do CITR, próximos a 0,2 , o que pode indicar que o mecanismo fisiológico responsável por reter o $\mathrm{Zn}$ nas raízes entrou em colapso, resultando em maior índice de translocação com doses elevadas deste elemento no solo, conforme observado na Figura 2C.

\section{CONCLUSÃO}

Doses próximas a $200 \mathrm{mg}$ de zinco $\mathrm{kg}^{-1}$ de solo argiloso estimulam a altura, diâmetro do colo, a massa seca aérea e radicular das mudas de timbaúva.

A timbaúva apresenta elevada tolerância ao zinco e por ser uma espécie arbórea nativa adaptada às condições ambientais do Brasil pode ser indicada para revegetação de áreas contaminadas com a aplicação de até $600 \mathrm{mg}$ de zinco $\mathrm{kg}^{-1}$ de solo argiloso. 


\section{AGRADECIMENTOS}

Ao Conselho Nacional de Desenvolvimento Científico e Tecnológico (CNPq), à Coordenação de Aperfeiçoamento de Pessoal de Nível Superior (Capes) pela concessão das bolsas, à Fundação Estadual de Pesquisa Agropecuária - FEPAGRO Florestas, unidade de Santa Maria (RS) e a FAPERGS.

\section{REFERÊNCIAS}

ABICHEQUIER, A. D.; BOHNEN, H. Eficiência de absorção, translocação e utilização de fósforo por variedades de trigo. Revista Brasileira de Ciência do Solo, Viçosa, v.22, p.21-26, 1998.

ARAÚJO, M. S. et al. Initial growth of African mahogany plants in response to zinc fertilization. African Journal of Agricultural Research, Adana, v. 12, p. 1022-1026, 2017.

BASSO, C. J. et al. Teores totais de metais pesados no solo após aplicação de dejeto líquido de suínos. Ciência Rural, Santa Maria, v. 42, p. 653-659, 2012.

BORGES JUNIOR, M. et al. Valores de referência local e avaliação da contaminação por Zn em solos adjacentes a áreas mineradas no município de Vazante-MG. Revista Brasileira de Ciência do Solo, Viçosa, v. 32, p. 2883-2893, 2008.

BROAdLeY, M. R. et al. Zinc in plants. Tansley review. New Phytologist, Lancaster, v. 173, p. 677-702, 2007.

BRUNETTO, G. et al. Frações de cobre e zinco em solos de vinhedos no Meio Oeste de Santa Catarina. Revista Brasileira de Engenharia Agrícola e Ambiental, Campina Grande, v. 18, n. 8, p. 805-810, 2014. CONSELHO NACIONAL DO MEIO AMBIENTE (Brasil). Resolução CONAMAn 420/2009. Disponível em: $<$ http://www.mma.gov.br/port/conama/legiab re.cfm?codlegi=620>. Acesso em: 15 abr. 2014.

EL-GHAMERY, A. A.; EL-KHOLY, M. A.; ABOU EL-YOUSSER, M. A. Evaluation of cytological effects of $\mathrm{Zn}^{2+}$ in relation to germination and root growth of Nigella sativa L. and Triticum aestivum L. Mutation Research, Massachusetts, v. 537, p. 29-41, 2003.

EMBRAPA Métodos de análises químicas para avaliação da fertilidade do solo. In: SILVA, F. C. (Org.). Manual de análises químicas de solos, plantas e fertilizantes. 2. ed. Brasília: EMBRAPA informação tecnológica, 2009.

EMBRAPA. Sistema brasileiro de classificação de solos. 3. ed. Brasília: SCT; EMBRAPA, 2013. 353 p. FERREIRA, D. F. SISVAR: a Computer Statistical Analysis System. Ciência e Agrotecnologia, Lavras, v. 35, p. 1039-1042, 2011.

GONÇALVES, J. L. M.; BENEDETTI, V. Nutrição e fertilização florestal. Piracicaba: [s. n.], 2005. $427 \mathrm{p}$.

GRINGS, M.; BRACK, P. Espécies Madeireiras. In: CORADIN, L.; SIMINSKI, A.; REIS, A. (Org.). Espécies nativas da flora brasileira de valor econômico atual ou potencial: plantas para o futuro Região Sul. 1. ed. Brasília: Ministério do Meio Ambiente, 2011. v. 1. p. 13-934.

HOODA, P. S. Trace elements in soils. 1st ed. United Kingdom: Wiley-Blackwell, 2010. 616 p.

INTERNATIONAL SOLID WASTE ASSOCIATION. Globalisation and waste management: Phase 1 concepts and facts. 2012. Disponível em: <http://www.iswa.org/en/79/the_international_solid_waste_ association.html>. Acesso em: 13 abr. 2014.

KABATA-PENDIAS, A. Trace elements in soils and plants. 4. ed. Boca Raton: CRC Press; Taylor \& Francis Group, 2010. 548 p.

KRÄMER, U. et al. Sucellular localization and apeciation of nickel in hyperaccumulator and non-accumulator Thlaspi species. Plant Physiology, Rockville, v. 122, p. 1343-1353, 2000.

LUX, A. et al. Differences in structure of adventitious roots in Salix clones with contrasting characteristics of cadmium accumulation and sensitivity. Physiologia Plantarum, Medford, v. 120, n. 4, p. 537-545, 2004. LUXRESEARCH. Technologies turn waste to profit. 2009. $11 \mathrm{p}$. Disponível em: $<$ http://luxresearch. web8.hubspot.com/Portals/86611/docs/research\%20downloads/lux_research_technologies_turn_waste_ to profit[1].pdf $>$. Acesso em: 13 abr. 2014.

MARQUES, T. C. L. L. S. M.; MOREIRA, F. M. S.; SIQUEIRA, J. O. Crescimento e teor de metais de mudas de espécies arbóreas cultivadas em solo contaminado com metais pesados. Pesquisa Agropecuária 
Brasileira, Brasília, v. 35, n. 1, p. 121-132, jan. 2000.

PAVLÍKOVÁA, D. et al. Glutamate kinase as a potential biomarker of heavy metal stress in plants. Ecotoxicology and Environmental Safety, New York, v. 70, p. 223-230, 2008.

PEREIRA, A. C. C. et al. Comportamento da Cordia africana Lam. cultivada em solo contaminado por metais pesados e tratado com materiais amenizantes. Ciência Florestal, Santa Maria, v. 23, n. 3, p. 329-336, jul./set. 2013.

PULFORD, I. D.; WATSON, C. Phytoremediation of heavy metal-contaminated land by trees, a review. Environment International, Lancaster, v. 29, p. 529-540, 2003.

RAJOO, K. S. et al. Heavy metal uptake and translocation Bydipterocarpus verrucosus from sewage sludge contaminated soil. American Journal of Environmental Sciences, Ontario, v. 9, n. 3, p. 259, 2013.

ROUT, G. R.; DIAS, P. Effect of metal toxicity on plant growth and metabolism: I. Zinc. In: LICHTFOUSE, E. et al. Sustainable agriculture. New York: Springer, 2009. p. 873-884.

SALT, D. E. Molecular analysis of plant adaptation to the environment. Dordrechtp: Kluwer Academic Publisher, 2001.

SANTOS, D. H. et al. Qualidade tecnológica da cana-de-açúcar sob adubação com torta de filtro enriquecida com fosfato solúvel. Revista Brasileira de Engenharia Agrícola e Ambiental, Campina Grande, v. 15, p. 443-449, 2011.

SILVA, R. F. et al. Comportamento de Peltophorum dubium (Sprengel) Taubert, Parapiptadenia rigida (Bentham) Brenan e Enterolobium contortisiliquum (Vell.) Morong Cultivadas em Solo Contaminado com Cobre. Ciência Florestal, Santa Maria, v. 21, p. 105-112, 2011.

SILVA, R. F. et al. Potencial da associação Pisolithus microcarpus com mudas. Ciência Florestal, Santa Maria, v. 26, n. 1, p. 181-191, 2016.

SOARES, C. R. F. S. et al. Toxidez de zinco no crescimento e nutrição de Eucalyptus maculat e Eucalyptus urophylla em solução nutritiva. Pesq. Agropececuária Brasileira, Brasília, v. 36, n. 2, p. 339348, 2001.

SOUSA, S. C. R. Tolerância aos metais pesados chumbo e zinco e potencial fitorremediador de mudas de espécies arbóreas. 2010. 85 f. Tese (Doutorado) - Universidade Estadual de Campinas, Campinas, 2010. TENNANT, D. A. Test of a modified line intersect method of estimating root length. Journal of Ecology, Oxford, v. 63, n. 3, p. 995-1001, nov. 1975.

TESTIATI, E. et al. Trace metal and metalloid contamination levels in soils and in two native plant species of a former industrial site: Evaluation of the phytostabilization potential. Journal of Hazardous Materials, New York, v. 248/249, p. 131-141, mar. 2013.

VAN SLYCKEN, S. et al. Safe use of metal-contaminated agricultural land by cultivation of energy maize (Zea mays). Environmental Pollution, Amherst, v. 178, p. 375-380, 2013.

ZACCHINI, M. et al. Metal tolerance, accumulation and translocation in poplar and willow clones treated with cadmium in hydroponics. Water Air and Soil Pollution, Ontário, v. 197, p. 23-34, 2009.

ZEITOUNI, C. F.; BERTON, R. S.; ABREU, C. A. Fitoextração de cádmio e zinco de um latossolo vermelho-amarelo contaminado com metais pesados. Bragantia, São Paulo, v. 66, n. 4, p. 649-657, 2007. 